\title{
Computerized Strength Training and Conditioning Programs
}

\author{
Eric L. Gossick, BS, CSCS, and Andrew C. Fry, PhD, CSCS \\ Exercise and Sport Science Laboratories \\ The University of Memphis
}

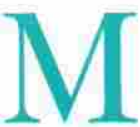

odern science has resulted in the growing use of computer technology to enhance an individual's work performance. Computerized sports training programs have become popular in recent years, especially strength training and conditioning computer programs.

These programs are designed to help strength and conditioning coordinators, coaches, athletic therapists, and personal trainers better serve their athletes, patients, and clients by developing effective strength training and conditioning regimens.

Coaches can make use of a computerized strength training and conditioning program to help them develop and monitor training regimens for numerous athletes from many teams in an effective and efficient manner. The variation of training necessary for an effective program can be simplified with the aid of a computer.

Changes in performance can also be effectively monitored and recorded, thus providing important information for the next training period. Compared to handwritten methods, an effective computerized strength and conditioning program allows the user to record and keep track of large numbers of athletes. Computers expedite the entire process and eliminate many of the mathematical errors that can occur with hand calculations.

Coaches can monitor the performance of their athletes at the beginning, middle, and end of a season or training period, with little or no trouble collecting, storing, and analyzing the data. They can design strength training and conditioning programs with greater detail since changing variables such as rest periods and training volumes and intensities is made much easier.

Athletic therapists who work in an environment that has no strength training and conditioning coach often must take on these additional responsibilities. A computerized training program can greatly facilitate this process, whether it is for rehabilitative purposes or is simply the general training program for healthy athletes.

An additional benefit of a computerized training program is that athletic therapists can record injuries and the effectiveness of the rehabilitation programs they are using. The rehab program can then be easily modified as the patient makes progress.

A library of effective rehabilitation programs can be developed to make for efficient use of time. These rehab programs can be easily tailored by changing the variables such as number of sets, type of muscle action used (concentric or eccentric), or the range of motion used.

The personal trainer's success depends on the progress of his or her clients. Personal trainers who use a computerized strength training and conditioning program would be better able to monitor progress. Progress is not simply limited to measures of strength but can also include changes in body composition or caloric expenditure, all of which can be charted in graphs and presented to the client.

Personal trainers could save time designing workouts and conducting progress tests by using such a program. These are just a few things that would make a personal trainer more professional in his or her approach to help clients achieve their goals.

\section{Periodization and Program Design}

A successful strength and conditioning program depends on proper use of the principles of periodization, or program variation. Periodization training principles have been adopted by strength and conditioning professionals around the world. The goal 
of periodization is to enhance human performance by avoiding overtraining and enhancing physiological adaptations. Periodization organizes training programs into cycles that have distinct goals and purposes.

In the classical concept, periodization is generally separated into three cycles: macro, meso, and micro. The macrocycle is generally a year in length but it can be any length that is more appropriate for the particular sport or activity. For example, an Olympic athlete may have a macrocycle that is 4 years long.

The mesocycle, the next cycle in the periodization theory, is generally weeks to months in length depending on the person's goals. For an athlete, the number and dates of competitions may dictate the number and length of the mesocyles used.

The shortest training cycle is the microcycle, which is often 1 week long.

Within each mesocycle there can be three distinct training periods: a preparatory period, a transitional period, and a competition period.

In addition, after every competition phase there is usually a period of active rest that helps keep the individual from becoming overtrained both physically and mentally. It typically consists of only light recreational activity. The preparatory period follows the active rest and prepares the athlete for competition.

Whether training athletes for maximal performance, helping to rehabilitate someone, or designing a program for general fitness in a healthy population, training variation is critical. Variation avoids the monotony and bore- dom that can occur over long periods. It also provides an everchanging stimulus to which the body must adapt.

Training stimulus in a strength program can be changed by manipulating five acute training variables: (a) choice of exercise, (b) order of exercises, (c) volume of training (i.e., total number of reps), (d) intensity of training (i.e., \% of maximum used), and (e) rest intervals between exercise sets.

If you consider all the possible combinations, it is easy to see there are literally thousands, many of which are very effective. The tricky part is to keep track of all these possibilities, and this is where a computerized program can come in handy.

In general it is important to remember that when strength training volume is high, training intensity must be low. Conversely, when training volume is low, training intensity can be high. Violation of this basic principle can easily result in overtraining and burnout. The use of an effective periodized program will help avoid these problems.

\section{Computer Technology- Advantages and Disadvantages}

The obvious advantage to computerized strength training and conditioning programs is the ability to do more in less time. When large numbers of athletes, clients, or patients are being trained, it is critical that the programs be tailored to each person's needs. Such customizing of training can involve any or all of the five acute training variables listed above.
Rather than calculating and designing the programs by hand, the training variables can be effectively manipulated by computer. Another advantage is the ability to store and save training and testing data.

"Rather than calculating and designing the programs by hand, the training variables can be effectively manipulated by computer. Another advantage is the ability to store and save training and testing data."

The disadvantages are few but noteworthy. One possible disadvantage is that an individual may rely too heavily on the computer program because of a lack of knowledge of strength training and conditioning theories and techniques. The individual might mistakenly believe that the program will make up for his or her lack of knowledge.

A computerized strength and conditioning program should not be viewed as a "black box" that does all the work for you. The operator must know something about program design. The resulting computer program is only as good at the information the operator puts into it. Instead of doing your thinking for you, it simply allows you to manage a large number of variables and programs effectively.

A lack of computer knowledge can also be a disadvantage for the user since it would prevent him or her from using the full program capabilities, or using them correctly. 


\section{Choosing the Right Computer Program}

The computer program you choose should meet your needs and make your job easier. Here are some of the program capabilities you should be aware of.

\section{Predesigned Workouts}

Does the program come with predesigned training progams? If so, how many? How many sports are represented (tennis, soccer, football, etc.)? Does the program have off-season, in-season, preseason training program variations? Can you change the exercises, sets, and repetitions in the predesigned workouts? You may like a particular program in general but might want to modify an existing program (e.g., use of only 2 sets for your new freshman soccer players instead of 4 sets).

Whenever predesigned programs are available, you must be able to critique them to determine whether they are really what you need. Do not assume that all programs are alike.

\section{Customized Programs}

Does the program allow you to design your own training regimen? Can you manipulate any of the acute training variables to provide the necessary variety? This is a critical consideration when selecting a computerized strength and conditioning program.

If you have little flexibility in designing your own programs, you may have difficulty satisfying your needs. Many software companies will have demonstration disks and supporting documentation to help you determine whether you can customize a program to fit your needs.

\section{Exercise Modality}

What type of exercise modality can the program monitor? Possibilities include weight training, plyometrics, speed training, aerobic training, flexibility, rehabilitation exercises, and others. This may be a major consideration if you are incorporating a variety of training stimuli into the total program.

It is important to remember that all of these training stresses accumulate to result in the overall stress to the system. Therefore the ability to incorporate additional exercise modalities into the computerized program increases the ability to monitor one's total exercise stress.

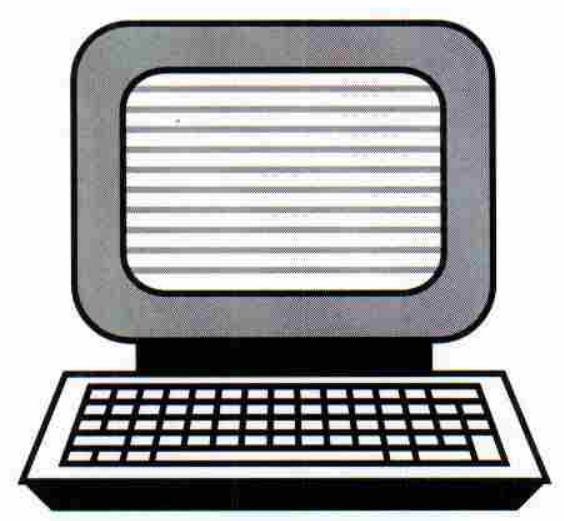

4. Monitoring the Acute Training Variables

Does the program monitor relative intensity (\% of 1-repetition $\max$ ), volume (total reps), and tonnage (reps $\times$ load)? Additional important variables may include mileage or distances (mileage per day, week, month), training times (times for the 40-yd dash, mile, etc.), or caloric expenditure (estimation of calories utilized during various types of exercise).

\section{Testing Capabilities}

Does the program support the collection and analysis of test results? Does it have preprogrammed strength tests (e.g., bench press or squat 1-RM), conditioning tests (e.g., 40-yd dash, agility runs), and fitness tests (e.g., sit and reach, body composition)? Does it give good instructions on how it administer these tests? Can it estimate the 1-RM from maximal efforts with multiple repetitions (e.g., 5- or 10-RM)? Can you print out the instructions and test results?

Can you add tests that you have developed or chosen to use for your particular needs? Are normative data or standards provided for these tests? If so, are the sources for these normative data provided? Are these norms based on scientific research, or are they simply provided by the manufacturer of the computer program?

\section{Organizational Abilities}

What kind of organizational abilities does it have? Can you program school dates, testing dates, holidays, or athletic competitions into it so you can design training programs around these dates? Can you see the training program in a monthly or weekly overview? Can you print out these overviews to post them on the wall of the weight room so athletes can see upcoming workouts?

How are training programs filed? Can you organize these programs by teams, athletic positions on teams, grade in school, age, etc.?

\section{Injury Records}

Can you keep a record of injuries? If so, does the program use standardized injury tracking guidelines? Is there a way to keep notes about the individual workout sessions such as whether or not the athlete had pain during the workout or had trouble finishing the 
workout? It is sometimes helpful to have a record of feedback from the attending clinician concerning particular injuries or illnesses that must be taken into account when developing an appropriate training program.

\section{Physiological/Medical Data}

Can you monitor physiological data such as blood pressure, anthropometric measures (e.g., girths), body weight (i.e., pre- and postexercise), body composition, and drug testing (dates and results of the tests)?

It is often helpful to keep a record of disease/disability information, including what medications are being taken and what medical conditions may be present (asthma, diabetes, etc.). Can you keep a record of the individual's personal physician and phone number, and an emergency contact person?

\section{Professional Guidelines}

Does the program furnish information on exercise guidelines provided by professional organizations such as the NSCA, ACSM, NATA, or similar organizations? Such guidelines can include exer- cise techniques (e.g., squat technique), aerobic prescription (frequency, duration, intensity), and injury treatment (e.g., the use of cryotherapy). Can these guidelines be printed out and given to the individual?

\section{Health Related Information}

Does the program provide information on health related issues such as an understanding of proper nutrition and other important issues?

\section{Graphs and Charts}

Can you graphically display strength increases, decreases in body fat, or other variables? Can they be printed out in graphs or charts that can be given to the individual? The axiom "a picture speaks a thousand words" certainly applies here, and a simple graph may be the best way to illustrate the effectiveness of a training program.

\section{Goals}

Can you record training goals, both short-term and long-term? Can you print them out to give to the individuals to help keep them focused?

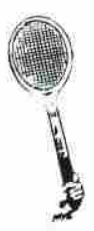

The U.S. Tennis Association and Society for Tennis Medicine \& Science

4th International Conference on Sports Medicine and Science in Tennis

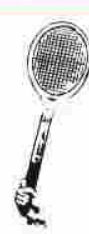

March 21-24, 1998, Biltmore Hotel, Coral Gables, FL

\section{Summary}

It is apparent that there many factors to consider when selecting a computerized strength and conditioning program. You must decide what features are important for your situation. Above all, in order to use a computerized program effectively, you must already be knowledgeable about how to design a proper program.

A computerized program is not meant to do the work for you. It is simply a method for managing a large number and variety of training programs. Many companies market computerized strength and conditioning programs. Listed below, but not in any preferential order, are a few to start you on your search.

Husker Power, Inc. $1-800-224-4044$

M-F Athletic Company, Inc.

Training Design Software Version 5.05 $1-800-682-6950$

The Fitness Buddy 1-800-895-5557

Power Systems, Inc.

Power 5.1 Strength Computer

Software

1-800-321-6975

Performance Software, Inc. Max Performer for Coaches 1-800-397-3332

B.S.D.I.

The Fitness Analyst 1-908-879-4991

Eric L. Gossick is completing his master's at the University of Memphis. He holds NSCA certification as a strength and conditioning specialist and is a USA Weightlifting certified club coach.

Andrew C. Fry is an assistant professor at the University of Memphis. He is a certified club coach with USA Weightlifting and chair of its sport science committee. He has a doctorate from Penn State. 